\title{
The Mass Function of Young Star Clusters in our Galaxy and Nearby Galaxies: Is It Universal?
}

\author{
Ram Sagar \\ State Observatory, Manora Peak, Nainital, 263129, Uttaranchal, India; \\ and Indian Institute of Astrophysics, Bangalore, 560 034, India
}

\begin{abstract}
Mass functions (MFs) derived from photometric observations of young star clusters of our Galaxy, the Magellanic Clouds (MCs), M31 and M33 have been used to investigate the question of universality of the initial mass function and presence of mass segregation in these systems. Observational determination of the MF slope of young star clusters have an inherent uncertainty of at least $\sim 1.0$ dex in the Milky Way and of $\sim 0.4$ dex in the MCs. There is no obvious dependence of the MF slope on either galactocentric distance or age of the young star clusters or on the spatial concentration of the stars formed or on the galactic characteristics including metallicity. Effects of mass segregation have been observed in a good number of young stellar groups of our Galaxy and MCs. As their ages are much smaller than their dynamical evolution times, star formation processes seem to be responsible for the observed mass segregation in them.
\end{abstract}

\section{Introduction}

The distribution of stellar mass at birth is defined as the initial mass function (IMF). Detailed knowledge of the IMF in different environments is valuable for studies that attempt to describe the spectral, photometric, and chemical evolution of integrated stellar systems because mass is one of the primary parameters that dictates the evolution of stars. Such studies are also used for constraining star formation theories and for understanding the early evolution of star clusters (Richtler 1994; Elmegreen 1999, 2000). The IMF also provides a valuable link between the easily observable population of luminous stars in a stellar system and the fainter, but dynamically more important low-mass stars. It is therefore important to know the true shape of the IMF in order to establish whether it is universal in time and space or whether it depends on parameters like metallicity, age, the properties of the natal molecular cloud or of the embedded young stellar population, etc. If the star formation is essentially a self-regulating process, then one might expect the IMF to be strictly universal. Alternatively, if stellar masses are determined only by the physical structure of the interstellar medium (e.g., fragmentation), then one may expect differences in the IMF that depend on local conditions such as cloud temperature, etc. In spite of many years of continued observations, answers are still unknown (Scalo 1998; Sagar 2000 and references therein). At first glance, it seems surprising in view of the simplicity 
of the concept of the IMF. However, after considering the steps involved in the observational determination of an IMF, one becomes aware of the large variety of difficulties and error sources involved in it. In order to emphasize this, some basic definitions are given below.

The stellar luminosity function, LF $\left(\equiv \phi\left(M_{i}\right)\right)$ represents the number of stars per unit magnitude interval in a given volume derived from observations in passband $i$ while the observed present day mass function, $\mathrm{MF}(\equiv N(M))$ denotes the number of stars per unit mass in a given volume. So they are related by

$$
N(M)=\phi\left(M_{i}\right) d M(z, \tau) / d M_{i},
$$

where $z$ refers to metallicity; $\tau=$ age/total main-sequence (MS) lifetime and $d M / d M_{i}$ is the MS mass - absolute magnitude relation for a passband $i$ which can be derived using theoretical stellar evolutionary and atmospheric models. The MF and IMF $(\equiv \xi(M, z, t \ldots))$ are related by

$$
N(M)=\int_{\max [0, T-t(M)]}^{T} \xi(M, z, t \ldots) \psi(t) d t,
$$

where $\psi(t)$ is the star formation rate (number of stars formed per unit time interval in a given volume); $T$ is the age of the galaxy being considered and $t(M)$ is the MS lifetime of a star with mass $M$. Therefore, the derivation of the IMF from the observed LF involves many steps. Consequently, determining the IMF using field stars is complicated because the population of field stars is a mixture of stars with different ages, distances, and metallicities (see Scalo 1986). Untangling these effects and correcting for the many biases inherent in the problem is an arduous task. In such work, the universality of the IMF (one of the questions) is assumed instead of being investigated and the shape of the IMF can only be estimated if further assumptions regarding the past star formation rate are made.

The behaviour of the IMF with time or metallicity or environment can be studied in a more direct way if one observes many star clusters with different ages, abundances, etc., because star clusters contain an (almost) coeval set of stars at the same distance with the same metallicity. Therefore, $\psi(t)$ in equation (2) can be replaced with $\psi_{c l} \delta\left(t-t_{0}\right)$; where $\psi_{c l}$ is the scale factor describing the number of stars formed in the cluster; $t_{0}$ is the cluster age and $\delta$ is the Dirac delta function. Equation (2) then becomes

$$
N(M, z, t \ldots)=\xi(M, z, t \ldots) \psi_{c l} .
$$

Therefore, in the simplest imaginable situation, the MF of a star cluster is equivalent to its IMF and it can in principle be determined from the LF using theoretical stellar evolutionary models. In reality the situation is different, because the stellar MF of any star cluster changes with time due to stellar as well as dynamical evolutionary effects. Such changes can, however, be ignored if one considers clusters younger than their dynamical evolution time which is taken as $100 \mathrm{Myr}$. We have therefore considered only star clusters younger than $100 \mathrm{Myr}$ in our further discussions and assumed that their present-day MF is equivalent to the IMF. Restricted to certain mass intervals, the IMF can be described by a power law in the form $\frac{d N}{d M}(=\xi(M)) \propto M^{-(1+x)}$, where $d N$ is the number of 
stars in the mass interval $d M$ at mass $M$. The MF slope is $x$. The Salpeter (1955) value of $x$ is 1.35 in the Solar neighbourhood.

As the evolutionary history of the MCs, M31, and M33 has been very different from that of our Galaxy, young star clusters of these galaxies are formed in different environments and there is also a wide range in metallicity and total mass of the system. A study of their present-day MF may therefore provide the answers to the questions raised above about the IMF. The present-day observed MFs of young star clusters in these galaxies are therefore discussed in the next section, while the sources of errors due to limitations in observations, procedures used to derive stellar MF and other results are described in the remaining part of the paper.

\section{Determination of the Cluster MF from Observations}

For the study of the MF in young star clusters and associations, deep and accurate photometric colour-magnitude diagrams (CMDs) of their members are essential. In recent years, modern CCD detectors and software for doing accurate photometry in crowded regions have made such observations possible. Due to better angular resolution capability, the Hubble Space Telescope (HST) has been able to resolve stars in extremely crowded regions like central regions of the MCs star clusters and outer regions of young stellar systems located in the nearby galaxies M31 and M33. This provides an opportunity for the study of stellar MF in young star clusters and associations in different galaxies of the Local Group.

Sagar (2000) has compiled reliable MFs determined for some young star clusters in our Galaxy. Such MFs determined recently by Pandey et al. (2001); Sagar, Munari \& de Boer (2001); Sanner \& Geffert (2001) and Sanner et al. (2001) have also been included in the present discussion. Fig. 1 shows the plot of MF slope $(x)$ versus galactocentric distance, $R_{G}$ and age of the clusters. The values of $x$, distance and age of the clusters have been taken from their respective photometric studies. In converting distance from geocentric to galactocentric in our Galaxy, the distance between the Sun and the Galactic centre is taken as $8.8 \mathrm{kpc}$. The range in $\mathrm{R}_{G}$ is from 6 to $12 \mathrm{kpc}$, while the ages range from 1 to 100 Myr. Reliable MF slope and cluster age for a number of young star clusters and associations located in MCs, M31 and M33 have also been determined recently using both groundbased telescopes and HST (see Table 1 in Sagar 2000). Lee et al. (2001) have presented the MF slopes of 6 extremely young star clusters in M33 using the HST observations. In Fig. 1, MF slopes of star clusters in the MCs and in M33 are plotted against age and $R_{G}$ respectively. The star clusters in the Large MC are located in different parts of the galaxy.

The values of $x$ seem to have no dependence on either $\mathrm{R}_{G}$ or cluster age in Fig. 1 and all are close to the Salpeter value. A few young clusters, however, seem to deviate significantly and there is a scatter of $\sigma_{x}=0.4$ dex around the Salpeter value. In M33, it appears that the value of $x$ increases with increasing $R_{G}$. However, more data points are needed to confirm it. Piskunov (1976) analyzed the MF slopes of 61 open star clusters and found the average value of $x=1.3 \pm 0.3$ in the mass range of 1 to $25 M_{\odot}$. Similarly, in five young and well populated open clusters, Sagar et al. (1986) derive an average value of $x=1.4 \pm 0.2$ within the mass range of $1.3-80 M_{\odot}$. Based on the CCD 

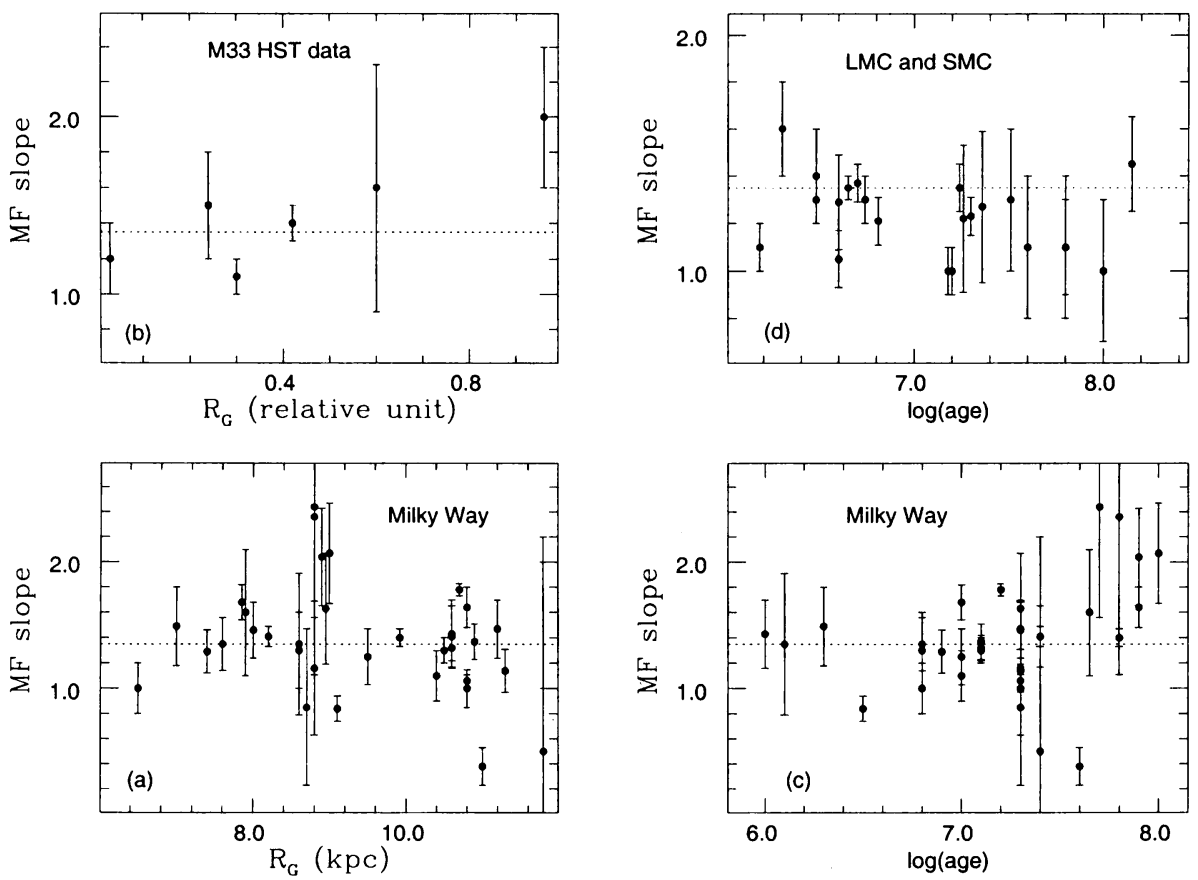

Figure 1. Plot of MF slope and its observational error against the galactocentric distance, $\mathrm{R}_{G}$ and age of the star clusters in the Milky Way in (a) and (c); in the MCs in (d) and in M33 in (b) part of the diagram. The horizontal dotted lines represent the Salpeter (1955) value of IMF slope in the Solar neighbourhood. Data for M33 clusters are taken from Lee et al. (2001). 
observations of 13 and 8 star clusters, Kjeldsen \& Frandsen (1991) and Phelps \& Janes (1993) derived average MF slopes of $1.3 \pm 0.2$ and $1.4 \pm 0.3$ respectively. For some more young star clusters, Subramaniam \& Sagar (1999) and Sanner $\&$ Geffert (2001) determined an average value of $x=1.4 \pm 0.3$ and $1.8 \pm 0.6$ respectively for the MF slope above $1 M_{\odot}$. Before we derive any conclusions from these and the plots in Fig. 1, it is necessary to know the accuracy of MF determinations in young star clusters, which is discussed below.

\section{Limitations in the observational determination of cluster MF}

The main factors which limit the precise determination of the young star cluster MF are scatter introduced by Poisson noise in a mass interval, the limited knowledge of accurate cluster parameters and stellar evolutionary models used to derive mass-luminosity relation, limited range in mass, data incompleteness and field star contamination, unresolved binaries and the dynamical and stellar evolution of the star clusters, etc. They are described briefly here.

\subsection{Poisson noise}

In the observed photometric CMDs, MS stars are identified and their LFs are determined. The inherently small number of stars in star clusters of our Galaxy results in large statistical uncertainties. Such errors are reduced significantly while studying the young MC star clusters as they are rich in stars. Recently, Kroupa (2001) has studied such errors using techniques of numerical simulations and found that in the MF slope determination of Galactic star cluster type objects, they introduce a scatter of $\sigma_{x} \sim 1$ above $1 M_{\odot}$. A similar study carried out by Sagar \& Richtler (1991) for MC star clusters indicates, as expected, a comparatively smaller value of $\sigma_{x} \sim 0.4$.

\subsection{Cluster parameters and MS mass-luminosity relation}

The accuracy of distance estimates is $\sim 15 \%$ while that of age determination is about $20 \%$ in young star clusters. The presence of non-uniform interstellar extinction has generally been noticed in young Galactic star clusters (Sagar 1987; Yadav \& Sagar 2001), which widen the cluster MS. It is therefore worthwhile to know whether it is present in clusters or not and if present, then its extent so that additional MS widening caused by it can be taken into account in the data analysis.

The effects of uncertainty in metallicity $(z)$ and of the MS mass-luminosity relations derived from the different theoretical stellar evolutionary models have been investigated by Sagar \& Richtler (1991). It is observed that the MF slope becomes flatter with decreasing value of $z$. The change in $x$ is $\sim 0.1$ and $\sim 0.3$ dex when $z$ varies from 0.02 to 0.004 and from 0.004 to 0.001 respectively. The $z=0.02$ stellar evolutionary models with convective core overshooting and without it can change the value of $x$ by $\sim 0.2$ dex which can be considered as error introduced due to uncertainty in mass-luminosity relation. 


\subsection{Data incompleteness and field star contamination}

In determining the MS cluster LF from the observed photometric CMDs, field star contamination and data incompleteness should be properly accounted for. Field star contamination increases with decreasing stellar brightness while data incompleteness increases with both increasing stellar crowding and decreasing stellar brightness. The procedures used for their corrections are described in detail earlier by Mateo (1988); Sagar \& Richtler (1991) and Banks et al. (1995) and recently by Sagar \& Griffiths (1998). These authors have also discussed the effects of improper correction of these parameters on the determination of the cluster MF slope. For example, it becomes flatter if correction for data incompleteness is not applied, while the lack of correction for field star contamination makes the MF steeper. Thus, one can say that, although both the corrections increase with decreasing stellar brightness, they affect the MF slope in exactly the opposite way. The change in MF slope can be significant, if the corrections are large.

\subsection{Dependence of MF slope on the mass loss rate}

In extremely young star clusters, mass loss occurs in massive $\left(>20 M_{\odot}\right)$ stars. The rate of mass loss can vary from one cluster to other and its effect on $x$ can be estimated as described below (Sagar et al. 1986).

Let us denote the initial mass of a star by $M_{b}$ and the mass determined from mass-luminosity relation by $M_{o}$. From the calculations of Maeder (1980), it is observed that at the end of MS evolution (where the effect of mass loss on mass estimate is at a maximum) the following relation is obtained:

$$
\log M_{o}=a \log M_{b}+b,
$$

where the constant $a$ is $<1$. If $\psi=\frac{d N}{d \log M_{o}}$ and $f=\frac{d N}{d \log M_{b}}$ are distribution functions of stars with masses $M_{o}$ and $M_{b}$ respectively, then it follows from equation (4) that $f=a \psi$. Further, if we define slopes of $\psi$ and $f$ as $x_{o}=-\frac{d \log \psi}{d \log M_{0}}$ and $x_{i}=-\frac{d \log f}{d \log M_{b}}$ respectively, we can easily find that $x_{b}=a x_{o}$, indicating the slope $x_{b}<x_{o}$ and depends on the value of $a$, which ultimately depends upon the rate of mass loss in massive stars. An analysis carried out by Sagar et al. (1986) for extremely young star clusters indicates that the value of $x$ will decrease with the increase in rate of mass loss. As the value of $a$ is $\sim 0.85$ (Maeder 1980), we conclude that by neglecting the effects of mass loss on MF determination, one can introduce a maximum error of $\sim 15 \%$ where mass loss is important (i.e., in stars with masses $\geq 20 M_{\odot}$ ).

\subsection{Effects of the coronal region in star clusters}

Recently, Nilakshi et al. (2002) have studied the spatial structure of the galactic open star clusters and find that a large fraction $(\geq 75 \%)$ of them have a coronal region surrounding the cluster core region, which is perceived directly by the eye as a star cluster. The annular width of the corona is generally more than twice the core radius. Due to their large area, coronal regions contain $\sim 70 \%$ of the cluster members. Their inclusion in the study of the cluster MF is important especially when effects of mass segregation are present in the clusters. As an example, we refer to the MF study of NGC 7654 by Pandey et al. (2001). 


\section{Mass segregation in young star clusters}

At the time of formation, if the star cluster had a uniform spatial stellar mass distribution, then as the cluster evolves dynamically, which leads to equipartition of kinetic energy in cluster members, the spatial stellar mass distribution changes and we would find the massive stars concentrated towards the centre of the cluster, as the low-mass stars attain high velocities and move away from the cluster centre. The mass segregation in a star cluster is then a result of dynamical evolution. The dynamical relaxation time, $\mathrm{T}_{e}$, is the time in which the individual stars exchange energies and their velocity distribution approaches a Maxwellian equilibrium. Numerical simulations of the early dynamical evolution of clusters containing a few hundred to a thousand stars indicate that dynamical mass segregation occurs on approximately the cluster relaxation time (see Bonnell \& Davies 1998 and references therein). They also indicate that dynamical evolution is more rapid (by an order of magnitude) than what $\mathrm{T}_{e}$ indicates, and the presence of a mass spectrum further accelerates the relaxation. In addition, the relaxation time depends upon the location in the cluster (see Meylan \& Heggie 1997 and references therein). How this affects the cluster structure and hence the dynamical process is not well understood. However, the simulations show that significant mass segregation among the heaviest stars in the core occurs in the local relaxation time but affecting a large fraction of the mass of the cluster requires a time comparable to the average relaxation time averaged over the inner half of the mass. The spatial distribution of members in young clusters (age $<<T_{e}$ ) cannot therefore be due to dynamical mass segregation and instead represents the primordial distribution. In some such young star clusters of our Galaxy as well as in the MCs, effects of mass segregation have been observed recently using ground based and HST photometric observations (Sagar et al. 1988; Pandey, Mahra \& Sagar 1992; Subramaniam, Sagar \& Bhatt 1993; Fisher et al. 1998; Richtler et al. 1998; Santiago et al. 2001 and references therein). The infrared images of star clusters still embedded in the natal dust/molecular clouds which make them opaque in visible light show that mass segregation could already be present at the very beginning of the cluster life (Lada \& Lada 1995). Such findings indicate that the assumption of no initial mass segregation used in most of the numerical simulations may not be justified, as they could lead to revision of the effects or time scale of the dynamical evolution.

\section{Discussion and Conclusions}

The inherent uncertainties discussed above clearly indicate that the observational determination of the MF slope of young star clusters and hence the IMF based on them, can never be determined more accurately than the scatter seen in Fig. 1 and also observed by other investigators. The variation in MF slope, if any, is less than the uncertainties discussed in section 4.

The young star clusters discussed here are situated in different parts of the MCs and nearby galaxies while the open clusters discussed are located in different regions of the Galactic disk. Even then, they seem to have MF slopes similar to the Salpeter value as the mean of $x$ is $1.38 \pm 0.45,1.33 \pm 0.04,1.23 \pm 0.17$ and $1.34 \pm 0.1$ for young star clusters of Milky Way, Small MC, Large MC and 
M33 respectively. The MF slopes of OB association NGC 206 in M31 determined from HST observations indicate a value of $1.4 \pm 0.5$ in the mass range $7-14 M_{\odot}$ (Hunter et al. 1996). The IMFs appear to have similar slopes in OB associations of the Milky Way, the LMC and the SMC, with a Salpeter-like slope 1.35 (cf. Massey, 1995a, b). Sakhibov \& Smirnov (2000) have studied the IMF in star formation complexes in 3 spirals, one irregular and one peculiar galaxy and conclude that the values of $x$, ranging from 1.06 to 1.57, are close to the Salpeter value. Meyer et al. (2000) have discussed the IMF in very young, partially embedded stellar clusters. They find that the mass distribution of young stars just emerging from different star forming molecular clouds are almost similar and are consistent with having been drawn from the Salpeter IMF. The low-mass $\left(<1 M_{\odot}\right)$ IMFs have recently been studied in young star clusters by Luhman et al. (2000). They are similar over a wide range of stellar densities and other environmental conditions. Using deep infrared surveys, Muench et al. (2000) derived the LF of two extremely young stellar clusters, IC 348 and NGC 2362, in the mass range $10-0.01 M_{\odot}$ and found that the underlying stellar MFs of these two star clusters are quite similar despite the differences in their apparent star formation rates and massive stellar contents. All these findings demonstrate that whatever it is that controls the star formation processes; there is no distinct difference between resulting MF slopes despite the difference of a factor of 10 in metallicity of these systems and supports the idea of some universal IMF as a consequence of star formation process in star clusters and associations. We therefore derive the following useful conclusions.

(1) The IMF does not vary wildly from region to region having different natal environments, metallicities, etc. though more subtle differences may exist.

(2) In some young star clusters, the observed mass segregation seems to be the imprint of star formation processes in them.

(3) The inherent uncertainty in the observational determination of MF slope of rich young star clusters like in the MCs is $\sim 0.4$ dex.

\section{References}

Banks, T., Dodd, R. J., \& Sullivan, D. J. 1995, MNRAS, 274, 1225

Bonnell, I. A., \& Davies, M. B. 1998, MNRAS, 295, 691

Elmegreen, B. G. 1999, ApJ, 515, 323

Elmegreen, B. G. 2000, ApJ, 539, 342

Fisher, P., Pryor, C., Murray, S., Mateo, M., \& Richtler, T. 1998, AJ, 115, 592

Hunter, D. A., Baum, W. A., O'Neil, E. J., \& Lynds, R. 1996, ApJ, 468, 633

Kjeldsen, H., \& Frandsen, S. 1991, A\&AS, 87, 119

Kroupa, P. 2001, MNRAS, 322, 231

Lada, E. A., \& Lada, C. J. 1995, AJ, 109, 1682

Lee, M. G., Park, H.S., Kim, S.C., Waller, W.H., Malumuth, E., Parker, J., \& Hodge, P. 2001, in IAU Symp 207, Extragalactic star clusters, eds. D. Geisler, E. K. Grebel \& D. Minniti (San Francisco: ASP) (in press) 
Luhman, K. L., Rieke, G. H., Young, E. T., Cotera, A. S., Chen, H., Rieke, M. J., Schneider G., \& Thompson, R. I. 2000, ApJ, 540, 1016

Maeder, A. 1980, A\&A, 92, 101

Massey, P., Lang, C. C., DeGioia-Eastwood, K., \& Garmany, C.D. 1995a, ApJ, 438,188

Massey, P., Johnson, K.E., \& DeGioia-Eastwood, K. 1995b, ApJ, 454, 151

Mateo, M. 1988, ApJ, 331, 261

Meyer, M.R., Adams, F.C., Hillenbrand, L.A., Carpenter, J.M., \& Larson, R.B. 2000, in Protostars and planets IV, eds. V. Mannings, A. P. Boss, \& S.

S. Russell (Tucson: The University of Arizona Press), 121

Meylan, G., \& Heggie, D. C. 1997, A\&AR, 8, 1

Muench, A. A., Lada, E. A., Alves, J. F., \& Lada, C. J. 2000, BAAS, 32, 1440

Nilakshi, Sagar, R., Pandey, A. K., \& Mohan, V. 2002, A\&A, 383, 153

Pandey, A. K., Mahra, H. S., \& Sagar, R. 1992, BASI, 20, 287

Pandey, A. K., Nilakshi, Ogura, K., Sagar, R., \& Tarusawa K. 2001, A\&A, 374, 504

Piskunov, A. E. 1976, Nauch.Inf., 22, 47

Phelps, R. L., \& Janes K. A. 1993, AJ, 106, 1870

Richtler, T. 1994, A\&A, 287, 517

Richtler, T., Fisher, P., Mateo, M., Pryor, C., \& Murray, S. 1998, in The Magellanic Clouds and Other Dwarf Galaxies, eds T. Richtler \& J.M. Braun (Shaker Verlag), 285

Sagar, R. 1987, MNRAS, 228, 483

Sagar, R. 2000, BASI, 28, 55

Sagar, R., \& Griffiths, W. K. 1998, MNRAS, 299, 777

Sagar, R., \& Richtler, T. 1991, A\&A, 250, 324

Sagar, R., Munari, U., \& de Boer K.S. 2001, MNRAS, 327, 23

Sagar, R., Myakutin, V. I., Piskunov, A. E., \& Dluzhnevskaya, O. B. 1988, MNRAS, 234, 831

Sagar, R., Piskunov, A. E., Myakutin, V. I., \& Joshi, U.C. 1986, MNRAS, 220, 383

Sakhibov, F. \& Smirnov, M. 2000, A\&A, 354, 802

Salpeter, E. E. 1955, ApJ, 121, 161

Sanner, J., \& Geffert, M. 2001, A\&A, 370, 87

Sanner, J., Brunzendorf, J., Will, J.-M., \& Geffert, M. 2001, A\&A, 369, 511

Santiago, B., Beaulieu, S., Johnson, R., \& Gilmore, G. F. 2001, A\&A, 369, 74

Scalo, J. M. 1986, Fund.Cosmic Phys., 11, 1

Scalo, J. M. 1998, ASP Conf. Ser. Vol. 142, The Stellar Initial Mass Function, eds. G. Gilmore \& D. Howell (San Francisco: ASP), 201

Subramaniam, A., \& Sagar, R. 1999, AJ, 117, 937

Subramaniam, A., Sagar, R., \& Bhatt, H. C. 1993, A\&A, 273, 100

Yadav, R. K. S., \& Sagar, R. 2001, MNRAS, 328, 370 


\section{Discussion}

B. Brandl: You said that the age of the cluster is much less than the dynamical timescales so that one observes the stars at their birth places. There are, however, two exceptions: First, the dynamical relaxation time is not a constant for all cluster stars but depends on their masses. It actually is much shorter for the most massive stars and their relaxation time could well be shorter than the cluster age. Secondly, expelling the gas in the very early stage of the cluster dramatically changes its gravitational potential and the least-bound stars - the very low mass stars - will leave the cluster. So one could observe a lack of low mass stars in the cluster center and a low mass star enhancement around the cluster, affecting the low end of the mass function.

R. de Grijs: HST observations of almost all compact star clusters, including the youngest ones, show at least some sign of mass segregation. This undoubtedly introduces a bias when comparing global mass functions. Can you comment on how mass segregation effects translate into uncertainties in these global mass function slopes?

R. Sagar: The uncertainties in the determination of global mass function slope of a cluster due to mass segregation depends upon the extent of radial coverage of the data. There is no effect, if the observations cover the entire cluster. However, global mass function slopes derived from data covering only part of the cluster are flatter than the actual slope, and the difference between them, which can be considered as the uncertainty introduced due to mass segregation, increases with decreasing coverage of the cluster radius.

C. Clarke: A comment on primordial mass segregation vs. rapid two-body relaxation. The only cluster where an unambiguous answer has so far been obtained is the Orion Nebula cluster, where Bonnell \& Davies showed through $\mathrm{N}$-body simulations that the central Trapezium of $\mathrm{O}$ stars could not be assembled through two-body relaxation within the $\sim 2 \mathrm{Myr}$ age of the cluster, and therefore argued strongly for primordial mass segregation in this case. 